\title{
Reducing Student Resistance to Active Learning: Applying Research Results to Faculty Development
}

\section{Lea K. Marlor, University of Michigan}

Lea Marlor is a Ph.D. student at the University of Michigan, studying Engineering Education Research. She joined the University of Michigan in Sept 2019.

Previously, she was the Associate Director of Education for the Center for Energy Efficient Electronics Science, a NSF-funded Science and Technology Center at the University of California, Berkeley. She managed undergraduate research programs to recruit and retain underrepresented students in science and engineering and also outreach to pre-college students to introduce them to science and engineering career opportunities. Ms. Marlor joined University of California, Berkeley in 2013. She has a B.S. in Materials Engineering from Rensselaer Polytechnic Institute.

\section{Dr. Cynthia J. Finelli, University of Michigan}

Dr. Cynthia Finelli is Professor of Electrical Engineering and Computer Science, Professor of Education, and Director and Graduate Chair for Engineering Education Research Programs at University of Michigan (U-M). Dr. Finelli is a fellow in the American Society of Engineering Education, a Deputy Editor of the Journal for Engineering Education, an Associate Editor of the IEEE Transactions on Education, and past chair of the Educational Research and Methods Division of ASEE. She founded the Center for Research on Learning and Teaching in Engineering at U-M in 2003 and served as its Director for 12 years. Prior to joining U-M, Dr. Finelli was the Richard L. Terrell Professor of Excellence in Teaching, founding director of the Center for Excellence in Teaching and Learning, and Associate Professor of Electrical Engineering at Kettering University.

Dr. Finelli's current research interests include student resistance to active learning, faculty adoption of evidence-based teaching practices, and the use of technology and innovative pedagogies on student learning and success. She also led a project to develop a taxonomy for the field of engineering education research, and she was part of a team that studied ethical decision-making in engineering students.

\section{Madison E. Andrews, University of Texas at Austin}

Madison Andrews is a STEM Education doctoral student, Mechanical Engineering master's student, and graduate research assistant for the Center for Engineering Education at the University of Texas at Austin. She received her B.S. in Mechanical Engineering from Clemson University in 2017.

\section{Ms. Bobbie Bermudez, University of Oregon}

Dr. Maura Borrego, University of Texas at Austin

Maura Borrego is Director of the Center for Engineering Education and Professor of Mechanical Engineering and STEM Education at the University of Texas at Austin. Dr. Borrego is Senior Associaate Editor for Journal of Women and Minorities in Science and Engineering. She previously served as Deputy Editor for Journal of Engineering Education, a Program Director at the National Science Foundation, on the board of the American Society for Engineering Education, and as an associate dean and director of interdisciplinary graduate programs. Her research awards include U.S. Presidential Early Career Award for Scientists and Engineers (PECASE), a National Science Foundation CAREER award, and two outstanding publication awards from the American Educational Research Association for her journal articles. All of Dr. Borrego's degrees are in Materials Science and Engineering. Her M.S. and Ph.D. are from Stanford University, and her B.S. is from University of Wisconsin-Madison.

Laura J. Carroll, University of Michigan

Nicholette Marie DeRosia, University of Oregon

Dr. Matthew Charles Graham

Dr. Jenefer Husman, University of Oregon 
Jenefer Husman received a doctoral degree in Educational Psychology from the University of Texas at Austin, in 1998. She served as an Assistant Professor at the University of Alabama from 1998 to 2002, when she moved to Arizona State University. In 2008 she was promoted by ASU to Associate Professor. She is currently an Associate Professor in the Educational Studies Department at the University of Oregon. Dr. Husman served as the Director of Education for the Quantum Energy and Sustainable Solar Technology Center - an NSF-funded Engineering Research Center from 2011-2016. Dr. Husman is an assistant editor of the Journal of Engineering Education, and is a member of the editorial board of Learning and Instruction. In 2006 she was awarded the U.S. National Science Foundation CAREER grant award and received the Presidential Early Career Award for Scientists and Engineers from the President of the United States. She has conducted and advised on educational research projects and grants in both the public and private sectors, and served as an external reviewer for doctoral dissertations outside the U.S. She publishes regularly in peer-reviewed journals and books. Dr. Husman was a founding member and first President of the Southwest Consortium for Innovative Psychology in Education and has held both elected and appointed offices in the American Psychological Association (APA) and the Motivation Special Interest Group of the European Association for Research on Learning and Instruction.

\section{Dr. Michael J. Prince, Bucknell University}

Dr. Michael Prince is a professor of chemical engineering at Bucknell University and co-director of the National Effective Teaching Institute. His research examines a range of engineering education topics, including how to assess and repair student misconceptions and how to increase the adoption of researchbased instructional strategies by college instructors and corporate trainers. He is actively engaged in presenting workshops on instructional design to both academic and corporate instructors. 


\title{
Reducing Student Resistance to Active Learning: Applying Research Results to Faculty Development
}

\begin{abstract}
Despite many studies confirming that active learning in STEM classrooms improves student outcomes, instructors' adoption of active learning has been surprisingly slow. This work-inprogress paper describes our broader research study in which we compare the efficacy of a traditional active learning workshop (AL) and an extended version of this workshop that also specifically highlights instructor strategies to reduce resistance (AL+) on instructors' beliefs about and actual adoption of active learning in undergraduate STEM classrooms. Through a randomized control trial (RCT), we aim to understand the ways in which these workshops influence instructors' motivation to adopt and the actual use of active learning. This RCT involves instructors and students at a large number of institutions including two-year college, four-year college, and large research institutions in three regions of the country and strategies to reduce student resistance to active learning. We have developed and piloted three instruments, which allow for triangulation of classroom data: an instructor survey, a student survey, and a classroom observation protocol. This work-in-progress paper will cover the current progress of our research study and present our research instruments.
\end{abstract}

\section{Introduction}

Past research has shown that instructors' use of active learning in the classroom can improve student learning, engagement, and interest in STEM; however, despite these findings, the translation of educational research to actual classrooms has been slow [1, 2, 3, 4]. Moreover, research suggests that the recurrent calls to increase the number, quality, and diversity of STEM graduates could, in fact, be substantially met if these evidence-based teaching practices were widely adopted in undergraduate STEM departments [5].

The primary challenge now is to increase the use of evidence-based teaching practices, specifically active learning, among STEM instructors. Previous research $[6,7,8]$ has identified instructor-reported barriers to the adoption of these practices, including concerns about: 1) the efficacy of the teaching practices; 2) the preparation time required to implement the teaching practices; 3 ) use of classroom time and corresponding concerns about covering all course content; and 4) student resistance. Previous research has thoroughly examined the first three of these concerns $[5,9,10,11,12,13,14]$ while, student resistance has not been systematically explored.

Here, we define student resistance as any negative behavioral response to active learning instruction that would discourage instructors from using that activity or active learning in the future. Examples include refusing to participate, vocally objecting in the class to doing the activity, giving low course evaluations, and/or distracting other students from participating. There are, however, effective strategies instructors can use to reduce student resistance $[15,16$, 17]. These strategies fall into three categories: planning, explanation, and facilitation [18]. Planning strategies can be used during the development of an activity, in how it is organized, and how to evaluate its effectiveness. Explanation strategies emphasize how an instructor can frame 
the purpose and the goal of the activity. Facilitation strategies focus on how to better engage students in the activity [18].

\section{Study Design}

Previous research has identified strategies for reducing student resistance, and in response to this research, we have created a multi-institutional team to train instructors on these strategies [18]. Specifically, we seek to: (1) design a faculty development workshop that can change instructor behavior by promoting adoption of active learning and use of strategies to reduce student resistance to active learning; (2) ascertain factors that impact the efficacy of the workshop; (3) examine how the use of specific strategies impacts student affective responses and student resistance to active learning; and (4) examine how student resistance to active learning influences instructors' future plans to use active learning in their courses.

\section{Research Questions}

This study seeks to answer the following research questions:

1. To what degree does a faculty development workshop $(\mathrm{AL}+)$ promote the use of planning, explanation, and facilitation strategies to reduce resistance?

2. To what extent does instructor motivation (i.e., self-efficacy and value) for adopting active learning moderate the effect of the workshop on instructor behavior?

3. To what extent does student resistance vary with the instructor's experience using active learning?

To answer our research questions, we developed a RCT study with the goal of determining if an intervention that teaches instructors how to mitigate this barrier would lead to greater adoption of active learning in STEM classrooms. The study can be broken down into four phases, with the first phase of participant recruitment and instrument development undertaken in Fall 2019. At three separate sites in the Midwest, Southwest, and West, we have recruited STEM instructors from 2-year and 4-year institutions to participate in our study. At each site, we aim to recruit a minimum of 75 participants who will be randomly assigned to one of three groups:

1) Active Learning only (AL): participants will attend a workshop that gives guidance only on active learning

2) Active Learning Plus (AL+): participants will attend a workshop that expands upon Workshop A to also include strategies for reducing student resistance to active learning

3) Control: participants do not attend a workshop during this study but will be offered an opportunity to attend a workshop in 2021, after the study is complete.

Once assigned to their groups, researchers will collect baseline data about participants in each group. This pre-intervention data collection includes faculty surveys, student surveys, and observations for a random subsample of classrooms in Winter/Spring 2020. Participants will receive an intervention workshop, or in the case of the control group, no intervention, in Summer 2020, followed by post-intervention data collection in Fall 2020. A timeline for this study can be found in Table 1. 
Table 1: Study Timeline

\begin{tabular}{|l|l|l|}
\hline Term & Phase & Activities \\
\hline Fall 2019 & Preparation & $\begin{array}{l}\text { Participant Recruitment and Instrument } \\
\text { Development }\end{array}$ \\
\hline Winter/Spring 2020 & Pre-Intervention & $\begin{array}{l}\text { Instructor Surveys, Student Surveys, and } \\
\text { Classroom Observations }\end{array}$ \\
\hline Summer 2020 & Intervention & $\begin{array}{l}\text { No Workshop - Control } \\
\text { Workshop A- Active Learning Only (AL) } \\
\text { Workshop B- Active Learning Plus (AL+) }\end{array}$ \\
\hline Fall 2020 & Post-Intervention & $\begin{array}{l}\text { Instructor Surveys, Student Surveys, and } \\
\text { Classroom Observations }\end{array}$ \\
\hline
\end{tabular}

\section{Phase 1- Preparation}

\subsection{Participant Recruitment}

Researchers in the Midwest, Southwest, and West are recruiting participants from colleges and universities within 150 miles of their respective campuses. This distance allows for in-person classroom observations by the researchers at each site. Participants were recruited via email, either to instructors directly or through department chairs when publicly listed on the institutions' website. Each regional site followed similar recruitment procedures and sent identical recruitment materials to reduce the variability between the sites.

Throughout this phase, we have recruited more than 300 instructors from over 105 schools across all three regions. Recruited instructors are teaching lecture-based STEM courses that are primarily taken by first- and/or second- year students. Particular attention was paid to ensure that instructors who are teaching at various institution types would be included in this study, including, community colleges, large research universities, minority serving institutions, and predominately white institutions.

In addition to recruiting instructors, we have collaborated with the IRB offices of these intuitions in order to ensure proper permissions are in place before data collection begins. We are currently in the process of finalizing IRB approval at sites and will report relevant statistics of how many participants we had to drop from this study as a result of institutional issues, as well as what types of institutions these instructors primarily teach at, in the final draft of this paper.

\subsection{Instruments}

The active learning approaches that instructors use, as well as student responses to those will be assessed through four different research methods: 1) instructor surveys given at the end of a class where active learning was used, 2) student surveys forwarded in an email after a class where active learning was used, 3) classroom observations, using a formal protocol, of the class session, and 4) pre- and post- workshop instructor surveys. All instructors, and their respective students, will be surveyed both pre- and post- intervention. Additionally, researchers will also travel to do in-person classroom observations for approximately 60 of these classrooms. These classrooms will be chosen at random with 20 observed in each of the three groups (AL, AL+, and control). The instruments are being built based on other validated instruments, including those that we 
have developed in our previous work [18]; however, since we are taking pieces from different surveys, we are doing additional validation with the surveys we build.

\section{Instructor Survey}

To assess instructors' perceptions of their use of active learning instruction, we designed a survey to measure instructors' use of active learning and their self-efficacy towards using it. Moreover, the survey was designed to identify perceived barriers instructors face when implementing active learning into their curriculum. The instructor survey measures 20 constructs with 99 total items and will focus on barriers, active learning use, planning strategies, explanation strategies, facilitation strategies, affective response, and behavioral response. Example items can be found in our previous work. [18]

\section{Student Survey}

In order to investigate students' perspectives of active learning instruction, we designed a survey to measure students' views of their instructor and how they perceived their use of active learning. Additionally, we will collect data that measures the students' engagement and perceived value of these activities. The student survey will measure ten constructs with 49 total items and will focus on active learning use, explanation strategies, facilitation strategies, affective response, behavioral response, belongingness, and self-efficacy. When possible, survey items were designed to correspond with the faculty survey items. Example items can be found in our previous work. [18]

\section{Observation Protocol}

Our observation protocol will document how often and for how long an instructor uses active learning within their classrooms. The observer will record information pertaining to how engaged the students are with any active learning activities and if there is any resistance occurring among the students. Additionally, the protocol will make note of how instructors are involved with the active learning activities and if they are using any of specific strategies to reduce student resistance to these activities.

\section{Phase 2- Pre-Intervention}

We have completed Phase 1 of this research and are beginning the pre-intervention data collection. Instructors will be assigned to treatment groups using stratified random sampling, based on their institution type as determined by the Carnegie classification. Once assigned to groups, we will administer the instructor and student surveys, as well as observe a random subset of the classes.

\section{Phase 3- The Intervention}

The AL workshop will focus on teaching instructors what active learning is and what the benefits are to using active learning in the classroom. After laying this groundwork, the workshop will delve into common concerns faculty have about using active learning in their classrooms, and strategies for successfully adopting active learning into their classrooms. The participants will experience active learning within the workshop, with the facilitator using it throughout the 
workshop, and they will also be given the opportunity to practice using active learning within the workshop.

The AL+ workshop will address everything that the AL workshop does, but it will also have an additional module that will focus on how faculty can reduce student resistance to active learning. The module will discuss why students resist active learning, as well research-based best strategies for overcoming resistance and will give instructors practice and feedback on using these strategies. We have created instruments in order to evaluate the planning, explanation, and facilitation strategies employed by instructors.

\section{Phase 4- Post-Intervention}

The same data collection as outlined in the pre-intervention section will occur post-intervention. All instructors and students will be surveyed, and again, 60 instructors will have an in-class observation. Through this data collection changes in instructor/student behavior from prior to the workshop are measured

\section{Future Work/Implications}

Student resistance is one of the key barriers cited by faculty against implementing active learning within their classrooms and helping faculty members overcome this barrier will hopefully lead to more adoption of active learning within their classrooms.

The results of our study will provide evidence-based support for whether student resistance is a measurable occurrence within classrooms and whether different strategies can be employed to reduce this resistance. The study also hopes to provide answers of if students are actually resisting active learning, as well as the instructors' perception of this resistance.

\section{Acknowledgments}

This material is based upon work supported by the National Science Foundation under Grant No DUE-1821488. Any opinions, findings, and conclusions or recommendations expressed in this material are those of the author(s) and do not necessarily reflect the views of the National Science Foundation. 


\section{References}

[1] Dancy, M., Henderson, C., \&; Turpen, C. (2016). How faculty learn about and implement research-based instructional strategies: The case of Peer Instruction. Physical Review Physics Education Research, 12(1), 010110.

[2] Gradinscak, M. (2011). Redesigning engineering education for a globalised world. International Journal of the Arts \&; Sciences, 4(25), 217-225.

[3] Jamieson, L. H., \&; Lohmann, J. R. (2012). Innovation with Impact: Creating a Culture for Scholarly and Systematic Innovation in Engineering Education. Washington, DC: American Society for Engineering Education.

[4] Stains, M., Harshman, J., Barker, M. K., Chasteen, S. V., Cole, R., DeChenne-Peters S. E., ... \&; Levis-Fitzgerald, M. (2018). Anatomy of STEM teaching in North American universities. Science, 359(6383), 1468-1470

[5] Freeman, S., Eddy, S. L., McDonough, M., Smith, M. K., Okoroafor, N., Jordt, H., \& Wenderoth, M. P. (2014). Active learning increases student performance in science, engineering, and mathematics. Proceedings of the National Academy of Sciences, 111(23), 8410-8415.

[6] Finelli, C., Richardson, K., \& Daly, S. (2013). Factors that influence faculty motivation of effective teaching practices in engineering. Paper presented at the ASEE Annual Conference and Exposition, Atlanta, GA.

[7] Froyd, J., Borrego, M., Cutler, S., Henderson, C., \& Prince, M. (2013). Estimates of use of research- based instructional strategies in core electrical or computer engineering courses. IEEE Transactions on Education, 56(4), 393-399.

[8] Prince, M., Borrego, M., Henderson, C., Cutler, S., \& Froyd, J. (2013). Use of research-based instructional strategies in core chemical engineering courses. Chemical Engineering Education, 47(1), 27-37.

[9] Felder, R. (1992). How about a quick one? Chemical Engineering Education, 26(1), 18-19.

[10] Felder, R. (1994). Any questions? Chemical Engineering Education, 28(3), 174-175.

[11] Felder, R., \& Brent, R. (1999). FAQs-2. Chemical Engineering Education, 33(4), 276-277.

[12] Felder, R., \& Brent, R. (2009). Active learning: An introduction. ASQ Higher Education Brief, 2(4), 1-5.

[13] Prince, M. (2004). Does active learning work? A review of the research. Journal of Engineering Education, 93, 223-232.

[14] Prince, M., \& Felder, R. (2006). Inductive teaching and learning methods: Definitions, comparisons, and research bases. Journal of Engineering Education, 95(2), 123-138.

[15] Finelli, C. J., Nguyen, K. A., DeMonbrun, R. M., Borrego, M., Prince, M. J., Husman, J., ... Waters, C. K. (2018). Reducing student resistance to active learning: Strategies for instructors. Journal of College Science Teaching, 47(5), 80-91.

[16] Nguyen, K. A., Husman, J., Borrego, M., Shekar, P., Prince, M. J., DeMonbrun, R. M., ... Waters, C. K. (2017). Students' expectations, types of instruction, and instructor strategies 
predicting student response to active learning. International Journal of Engineering Education, 33(1A), 2-18.

[17] Tharayil, S. A., Borrego, M., Prince, M., Nguyen, K. A., Shekhar, P., Finelli, C. J., \&;

Waters, C. K. (2018). Strategies to mitigate student resistance to active learning. International Journal of STEM Education, 5(7), 1-16.

[18] Finelli, C.J., Carroll, L., Prince, M. \& Husman, J. (2019). Promoting adoption of active learning and use of strategies to reduce student resistance to active learning. Research in Engineering Education Symposium. Retrieved from http://par.nsf.gov/biblio/10112911 